\title{
Des histoires familiales pour engager les élèves dans l'écrit et valoriser les langues et cultures d'origine à l'école: les « textes identitaires plurilingues »
}

\author{
Par Angela Stoica, enseignante-chercheuse \\ Chargée de cours en Didactique du français langue seconde \\ à l'Université de Montréal
}

Résumé: Cet article présente un projet de recherche-action intitulé Écriture et histoires familiales de migration, une recherche action pour promouvoir les compétences à écrire des élèves allophones immigrants et réfugiés dans les écoles primaires et secondaires du Québec (FQRSC, Vatz-Laaroussi, Armand, et al. 2010-2013). Le projet avait pour but de développer les compétences à l'écrit en français des jeunes élèves allophones immigrants durant leurs premières années de scolarisation en pays d'accueil. Pour ce faire, nous avons croisé l'histoire familiale et les compétences en écriture, en invitant les familles à participer au projet, accompagnées par des organismes communautaires. Ce projet visait la production d'un livre de leur histoire familiale de migration. Ce livre, personnel à chaque jeune, conçu et réalisé en classe, est écrit en français, avec des apports de membres de leur famille et éventuellement de leur langue d'origine.

\section{1 - Problématique}

Le Québec accueille chaque année près de 50000 immigrants indépendants et réfugiés. De plus en plus, ces nouveaux arrivants proviennent de toutes les régions $\mathrm{du}$ monde et une grande majorité d'entre eux est allophone. Diverses recherches concernant les élèves immigrants et réfugiés allophones et leur performance scolaire démontrent que l'apprentissage de la langue française représente pour eux un premier facteur de retard dans la trajectoire scolaire. (Armand, 2005; Steinbach, 2008). C'est au cours des deux premières années de scolarisation au Québec que cet obstacle est le plus manifeste, soit au primaire ou au secondaire, essentiellement dans les classes d'accueil (Allen, 2006), mais les effets du manque de maîtrise de langage scolaire continuent durant les études postsecondaires (Roessingh \& Kover, 2003). Plusieurs de ces élèves, en particulier les réfugiés, ont aussi eu une trajectoire scolaire perturbée et certains présentent un grand retard scolaire voire ne sont jamais allés à l'école.

Selon le milieu social dont on provient, la culture dans laquelle on vit, la trajectoire qu'on a suivie, la langue dans laquelle on écrit, l'écriture prend des sens différents. Il $\mathrm{y}$ a un lien entre littératie et culture (Painchaud et al., 1994) et les stratégies d'enseignement de l'écrit doivent être différenciées selon le type d'apprenants et leur culture. Les enfants allophones nouvellement arrivés, lorsqu'ils rentrent à l'école québécoise, apportent aussi tout un bagage à la fois cognitif, culturel et affectif qui est lié à leur parcours migratoire. Leurs expériences de scolarité avant le départ du pays d'origine et aussi des repères culturels, des événements familiaux, parfois des traumatismes dus à l'exil viennent donner à la langue, à l'école, au français et à l'écrit des sens nouveaux (Rousseau, 2005). Les élèves immigrants allophones ont le double défi de développer des compétences à l'écrit dans une langue seconde, parfois sans la littératie dans leur langue maternelle 
(Armand, 2007). Dès lors les enfants sous ou non scolarisés dans leur pays d'origine ou au cours de leur trajectoire migratoire comme les réfugiés et en particulier ceux qui ont vécu dans des camps durant plusieurs années, rencontreront des difficultés supplémentaires dans l'apprentissage du français écrit.

2- Hypothèse et questions de recherche

Nous avons posé l'hypothèse que l'écriture de l'histoire familiale de migration représente un contexte significatif qui peut aider le jeune élève immigrant allophone à développer des apprentissages en écriture et en français dans un processus gratifiant, ludique et authentique.

Notre projet de recherche action a permis de répondre aux questions suivantes: 1) Comment favoriser l'écriture de l'histoire familiale en milieu scolaire pour développer la motivation à l'apprentissage de l'écrit et du français tout en reconnaissant la valeur symbolique et affective de la langue d'origine et des savoirs familiaux ? 2) Comment faire participer la famille à cette écriture? 3) Quels outils pédagogiques et didactiques utiliser pour que cette écriture de l'histoire familiale devienne une motivation pour l'apprentissage du Français et de l'écriture? 4) Quels outils construire pour que dans cette expérimentation les élèves développent leurs compétences à écrire en français?

3- Les objectifs de la recherche action : ils sont de 5 ordres.

1) Objectifs de connaissance : Apporter de nouvelles connaissances sur les motivations à l'écriture, sur les collaborations familleécole et sur les questions linguistiques pour des jeunes immigrants et réfugiés allophones à l'école primaire et secondaire québécoise durant leurs deux premières années au Québec.

2) Objectifs d'action: Construire, expérimenter et évaluer l'implantation d'un projet portant sur l'écriture d'histoires familiales de migration en milieu scolaire primaire et secondaire par les jeunes et leur famille.

3) Objectifs de formation et de transfert: Former des enseignants par l'expérimentation, transférer ces nouveaux savoirs et ce projet pilote à d'autres milieux scolaires et communautaires.

4) Objectifs de transformation: Transformer les pratiques enseignantes, les collaborations famille-école et les représentations mutuelles.

5) Objectifs de développement éthique: Développer une réflexion éthique sur les rôles de l'école, des familles et des organismes communautaires pour les apprentissages en écriture et en français des jeunes.

\section{Partie B: Pistes de solution}

1- Des résultats utiles pour divers publics

Notre projet de recherche action repose sur l'hypothèse que l'écriture de l'histoire familiale en milieu scolaire, en français avec des « clins d'œil » à la langue d'origine, avec des apports de la famille et l'aide du milieu communautaire, représente un processus de motivation à l'apprentissage du français écrit pour les jeunes immigrants. C'est aussi un processus qui permet la construction identitaire du jeune et son adaptation à son nouveau milieu. Finalement il s'agit au travers de cette action d'écriture de l'histoire familiale en milieu scolaire de favoriser une représentation positive à la fois de la langue française et de l'écriture. Aussi les résultats et retombées de notre projet visent comme indiqué dans les objectifs, plusieurs publics représentés dans nos partenaires.

Le milieu académique et de la recherche bénéficie des résultats de la recherche action à trois niveaux : 1) L'identification des dimensions d'un contexte significatif favorisant la motivation des jeunes élèves allophones pour apprendre à écrire en français comme l'histoire familiale, la trajectoire de migration, les souvenirs, les réseaux, la langue maternelle et les talents 
déjà développés. 2) Une analyse des représentations des acteurs scolaires et immigrants quant aux langues (française et d'origine), quant à l'écriture et quant à l'histoire familiale. 3) L'enrichissement des connaissances sur les pratiques pédagogiques pertinentes et novatrices visant l'écriture authentique en contexte significatif pour ces jeunes (en particulier, les stratégies de différenciation et de personnalisation des apprentissages ressortent ici comme des conditions de réussite. Évidemment ces résultats ont des impacts directs pour les milieux de pratique. Les commissions scolaires, les milieux scolaires et les enseignantes bénéficient de l'expérimentation et des stratégies pédagogiques innovantes qui y sont développées ainsi que de la réflexion pédagogique et éthique qui y est menée. On envisage un enrichissement des pratiques pédagogiques en situation d'accueil et d'apprentissage du français langue seconde. Les familles et les jeunes bénéficient directement de l'expérimentation pour les enfants qui y ont participé. Les parents vont ainsi se rapprocher de l'école, mieux connaître le système dans lequel s'inscrit leur enfant et comment ils peuvent l'accompagner. Dans le même temps le personnel scolaire connaît mieux ses élèves, leur trajectoire et leurs proches, développant ainsi de nouvelles collaborations familles-écoles. $\mathrm{La}$ participation des organismes communautaires au projet permet ce rapprochement entre les familles et l'école et ouvre sur de nouveaux partenariats entre l'école et le milieu de vie, favorisant ainsi une école inclusive. Finalement les décideurs et gestionnaires peuvent bénéficier de ces résultats pour améliorer la formation des enseignants en classes d'accueil et pour mieux gérer les publics de ces classes. De manière générale, ce projet représente un prototype des actions qui peuvent être menées en classe en collaboration avec les familles et les milieux, pour développer les apprentissages en écriture en français et renforcer la motivation à apprendre et à écrire des jeunes.

3- Grands principes issus du projet et retombées

La recherche action fait ressortir 4 grands principes qui sont illustrés dans les outils issus de la recherche action et qui peuvent servir de fondements à de nouveaux projets: 1) Motiver grâce à des situations signifiantes. 2) Valoriser le dialogue entre les langues. 3) Faire de la place au dialogue entre les familles et l'école. 4) Oser des pratiques innovantes. Ainsi un guide d'accompagnement des enseignantes et milieux communautaires souhaitant mettre en œuvre de tels projets, a été produit ainsi que deux DVD qui enrichissent les données pédagogiques et didactiques issues de la recherche action. Des formations et ateliers peuvent être donnés dans les commissions scolaires et auprès des conseillers pédagogiques. Des fiches pédagogiques pour les ateliers en classe sont aussi disponibles pour les enseignantes ainsi qu'une boîte à outils identifiant des articles, sites WEB, vidéos pertinents à la mise en œuvre de projets d'écriture signifiants ainsi que des outils utilisables directement en classe.

\section{Bibliographie}

Armand, F., Le, T. H., Combes, É., Saboundjian, R. et Thamin, N. (2011). L'enseignement de l'écriture en langue seconde Département de didactique, Université de Montréal.

Armand, F. Dagenais, D. et Nicollin, L. (2008). La dimension linguistique des enjeux interculturels : de l'Éveil aux langues à l'éducation plurilingue. Revue Éducation et Francophonie, vol. XXXVI, no $1: 44-64$.

Cummins, J., \& Early, M. (2011). Identity texts : the collaborative creation of power in multilingual schools. Stoke on Trent ; Sterling, Va.: Trentham Books.

Kanouté, F. et Vatz-Laaroussi, M., (éd.) (2008). Relations écoles-familles de 
minorités ethnoculturelles [numéro thématique]. Revue des sciences de l'éducation, 34(2).

Rachédi, L., Guesdon, N. (2011). Ateliers récits identitaires, promotion des histoires familiales de jeunes immigrants en maison de jeunes. École de travail social (UQÀM), 59 pages.

Rachédi, L., Pierre, A. (2007). « Historioriser " l'immigration ou comment accompagner les familles immigrantes en partageant leur histoire ». Revue de l'Association des psychothérapeutes familiaux et conjugaux du Québec, vol. 32, no 2, p. 4-13.

Taylor, Bernhard, Garg et Cummins (2008). « Affirming Plural Belonging: Building on Students' Family-Based Cultural and Linguistic Capital through Multiliteracies Pedagogy ». Journal of Early Childhood Literacy, 8(3), 269-294.

Vatz Laaroussi M.(2009). Mobilités, réseaux et résilience : le cas des familles immigrantes et réfugiées au Québec, PUQ, Collection Problèmes sociaux et intervention sociale, $250 \mathrm{p}$.

Sitographie

Site d'activités Éveil au langage et ouverture à la diversité linguistique

ÉLODIL: www.elodil.com

Site du CEETUM :

http://www.ceetum.umontreal.ca/fr/actualit

es/pub-a-

signaler/publication/article/litterature-

jeunesse-bibliographie-selective-s/ 